\section{Tools for metabolomics}

New tools are filling the gap in our metabolomics capabilities.

Metabolomics can provide a better understanding of the state of cellular and biological processes at different stages of growth, under disease conditions, in response to stimuli, and even as a molecular signature for cell identification. The metabolome is thought to be the closest representation of phenotype and shows tremendous potential in applications such as understanding cellular processes in both normal and diseased tissues, environmental research, agriculture, biomarker discovery and personalized medicine. In spite of this, however, comprehensive data analysis and identification of the complex metabolite pool is still a challenge.

One limitation is the sheer number of still uncharacterized metabolites the small sizes of publically available spectral libraries, in comparison to the possible metabolite space, means that a high percentage of peaks remain unidentified in mass-spectrometrybased experiments. Another limitation is accurate statistical validation for largescale spectral assignments. Again, due to the high metabolite diversity, generation of a comprehensive decoy set to estimate

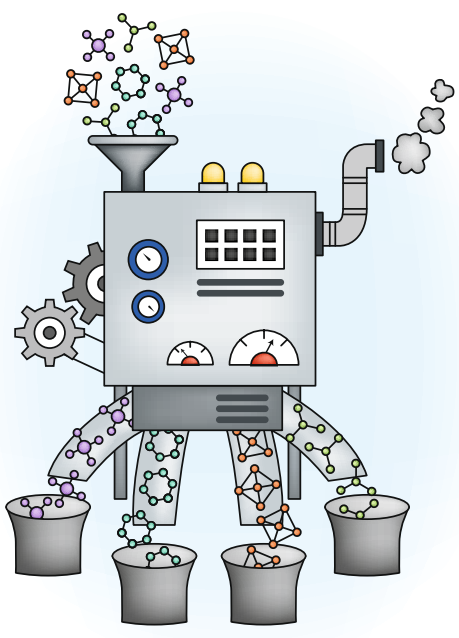

Sophisticated tools are needed to process metabolomics data. Credit: Kimberly Caesar/ Springer Nature

the false discovery rate, which has been successful in the field of proteomics, is not feasible. Available approaches often require parameters to be adjusted for each data set (Nat. Commun. 8, 1494, 2017).

However, progress is being made. Some of the new approaches for compound identification include integration of
CSI:FingerID in SIRIUS4 (Nat. Methods 16, 299-302, 2019) for searching molecular structure databases, multi-level annotations using chemoinformatics strategies for carbon number determination and metabolite classification (Nat. Methods 16, 295-298, 2019), integrative metabolomics platforms such as MetaboAnalyst 4.0 (Nucleic Acids Res. 46, W486-W494, 2018), and metabolic reaction network-based annotation (Nat. Commun. 10, 1516, 2019). Creative applications and analyses include the use of metagenomics and genomescale metabolic models to aid microbiome modeling (Nat. Microbiol. 3, 456-460, 2018) and integration of metabolomics data with metabolic pathways for identifying metabolites affecting particular phenotypes (Nat. Biotechnol. 36, 316-320, 2018).

The potential applications of metabolomics are only beginning to be fully explored. As researchers combine other -omics data and with more sophisticated study designs, the need for methods, both generalizable and specialized, will continue to grow. We look forward to further developments in the field.

\section{Arunima Singh}

Published online: 6 January 2020

https://doi.org/10.1038/s41592-019-0710-6

\section{Putting on the squeeze}

High-throughput methods for measuring mechanical properties of cells are ready for prime time.

The cytoskeleton, cell membrane, cytoplasm and embedded organelles give rise to the mechanical properties of cells, and these properties inform cellular state and functions. Changes in biomechanical properties can go hand in hand with developmental changes or even transformations of healthy cells into cancer cells.

Traditional methods for assessing the viscoelastic properties of cells include atomic force microscopy, optical tweezers or micropipette aspiration. However, these approaches can only be applied to one cell at a time, and throughput is therefore typically low - on the order of tens to hundreds of cells per hour. In recent years, a variety of alternatives to these approaches have emerged that can be classified under the umbrella of deformability cytometry (for example, Proc. Natl Acad. Sci. 109 7630-7635, 2012; Proc. Natl Acad. Sci. 110, 7580-7585, 2013; Nat. Methods 12,

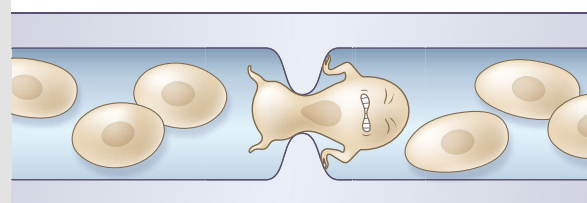

properties of individual cells. As the various forms of deformability cytometry operate with different geometries and under different stress and strain rates, the techniques may be sensitive to different aspects of a cell's biomechanics. However, this is no different from the more traditional methods (Nat. Methods

Cell squeezing through a constriction in a microfluidic channel. Credit: Debbie Maizels/ Springer Nature

199-202, 2015). These new kids on the block are mainly based on microfluidic approaches, and they can reach a throughput of thousands to millions of cells per hour.

In one class of approaches, cells squeeze through constrictions in microfluidic channels, and the cells' time to pass through the constriction correlates with the mechanical properties of the cells. Other approaches are contactless and induce deformations through flow and/or pressure gradients while deformations are read out via high-speed imaging.

Deformability cytometry approaches measure the cumulative viscoelastic 15, 491-498, 2018); the choice of method needs to be informed by the experimental question. More importantly, these differences may offer opportunities to gain insights into the underlying principle of cellular mechanics.

Microfluidics-based approaches are more accessible than the traditional approaches for measuring mechanical properties, and several deformability cytometry methods have been or are being commercialized. We expect to see uptake of these approaches in the broader community and look forward to their application to a wide range of questions.

Nina Vogt

Published online: 6 January 2020

https://doi.org/10.1038/s41592-019-0711-5 